\title{
The augmented base locus of real divisors over arbitrary fields
}

\author{
Caucher Birkar
}

\begin{abstract}
We show that the augmented base locus coincides with the exceptional locus (i.e. null locus) for any nef $\mathbb{R}$-Cartier divisor on any scheme projective over a field (of any characteristic). Next we prove a semi-ampleness criterion in terms of the exceptional locus generalizing a result of Keel. We also discuss some problems related to augmented base loci of log divisors.
\end{abstract}

\section{INTRODUCTION}

The base locus of a linear system is a fundamental notion in algebraic and especially birational geometry. The restricted base locus (also called the nonnef locus) and the augmented base locus (also called the non-ample locus) are refinements of the base locus which capture more essential properties of divisors and linear systems. These are closely related to important concepts and problems in birational geometry, eg see [4],[16],[15],[9],[8],[6],[2].

We start with some definitions.

The augmented base locus. Let $X$ be a scheme. An $\mathbb{R}$-Cartier divisor is an element of $\operatorname{Div}(X) \otimes_{\mathbb{Z}} \mathbb{R}$ where $\operatorname{Div}(X)$ is the group of Cartier divisors. A $\mathbb{Q}$-Cartier divisor is defined similarly by tensoring with $\mathbb{Q}$.

Definition 1.1 Let $X$ be a projective scheme over a field $k$. The stable base locus of a $\mathbb{Q}$-Cartier divisor $L$ is defined as

$$
\mathbf{B}(L)=\bigcap_{m \in \mathbb{N},} m L \text { Cartier } \operatorname{Bs}|m L|
$$

that is, it is the set of those points $x \in X$ such that every section of every $m L$ vanishes where $m$ is a positive integer and $m L$ is Cartier. The base locus, stable base locus, and all the other base loci defined below are considered with the reduced induced structure. The augmented base locus of $L$ is defined as

$$
\mathbf{B}_{+}(L)=\bigcap_{m \in \mathbb{N}} \mathbf{B}(m L-A)
$$

where $A$ is any ample Cartier divisor.

The augmented base locus of $\mathbb{R}$-Cartier divisors on smooth projective varieties was defined in [8]. For basic properties of the augmented base locus in this

Date: December 15, 2015.

2010 MSC: 14E30, 14A15. 
context see [9][8]. We give a different definition which is more convenient for our purposes (the two definitions agree, by Lemma 3.1 (3) below).

Definition 1.2 Let $X$ be a projective scheme over a field $k$. Let $L$ be an $\mathbb{R}$-Cartier divisor on $X$. We can write $L \sim_{\mathbb{R}} \sum t_{i} A_{i}$ where $A_{1}, \ldots, A_{r}$ are very ample Cartier divisors and $t_{i} \in \mathbb{R}$. The $A_{i}$ are not necessarily distinct and the expression is obviously not unique. Define $\langle m L\rangle=\sum\left\lfloor m t_{i}\right\rfloor A_{i}$ which depends on the above expression. Next define the augmented base locus of $L$ as

$$
\mathbf{B}_{+}(L)=\bigcap_{m \in \mathbb{N}} \mathbf{B}(\langle m L\rangle-A)
$$

where $A$ is any ample Cartier divisor.

It turns out that $\mathbf{B}_{+}(L)$ does not depend on the choice of $A$ nor the $A_{i}$ nor the expression $L \sim_{\mathbb{R}} \sum t_{i} A_{i}$ (see Lemma 3.1). In particular, $\mathbf{B}_{+}(L)$ depends only on the $\mathbb{R}$-linear equivalence class of $L$.

Relation with the exceptional locus. Before stating our first result we recall the definition of exceptional locus.

Definition 1.3 Let $X$ be a projective scheme over a field $k$ and $L$ an $\mathbb{R}$-Cartier divisor on $X$. The exceptional locus of $L$ (also called the null locus when $L$ is nef) is defined as

$$
\mathbb{E}(L):=\bigcup_{\left.L\right|_{V} \text { not big }} V
$$

where the union runs over the integral subschemes $V \subseteq X$ with positive dimension.

Theorem 1.4. Let $X$ be a projective scheme over a field $k$. Assume that $L$ is a nef $\mathbb{R}$-Cartier divisor with a given expression $L \sim_{\mathbb{R}} \sum t_{i} A_{i}$ as in 1.2, and that $A$ is a very ample Cartier divisor on $X$. Then

$$
\mathbf{B}_{+}(L)=\mathbf{B}(\langle n L\rangle-A)=\mathrm{Bs}|\langle n L\rangle-A|=\mathbb{E}(L)
$$

for any sufficiently divisible $n \in \mathbb{N}$.

The theorem was first proved for $X$ smooth, $\mathbb{Q}$-Cartier $L$, and $k$ algebraically closed of characteristic zero by Nakamaye [16] using Kodaira type vanishing theorems, and this was generalized to $\mathbb{R}$-Cartier divisors by Ein-LazarsfeldMustaţă-Nakamaye-Popa [8]. Nakamaye's result was extended to log canonical varieties by Cacciola-Lopez [5] again by using Kodaira type vanishing theorems. They also give some applications to the moduli spaces of curves. Related results concerning the restricted volume are proved on normal varieties by BoucksomCacciola-Lopez [3].

The theorem was proved by Cascini-McKernan-Mustaţă [6] when $k$ is algebraically closed of positive characteristic using techniques related to Keel [12]: the main ingredients are Serre vanishing and the Frobenius. Fujino-Tanaka [10] employ similar arguments on surfaces using Fujita vanishing and the Frobenius. 


\section{The augmented base locus of real divisors over arbitrary fields 3}

We will also use Fujita vanishing but not the Frobenius.

A semi-ampleness criterion. The following semi-ampleness result was first proved by Keel [12] when $k$ has positive characteristic. A simplified proof of Keel's result was given by Cascini-McKernan-Mustaţă [6].

Theorem 1.5. Let $X$ be a projective scheme over a field $k$. Assume that $L$ is a nef $\mathbb{Q}$-Cartier divisor on $X$. Then there is a closed subscheme $Z \subseteq X$ such that

- the reduced induced scheme associated to $Z$ is equal to $\mathbb{E}(L)$, and

- $L$ is semi-ample if and only if $\left.L\right|_{Z}$ is semi-ample.

When $k$ has positive characteristic we can use the Frobenius to show that in fact we can take $Z=\mathbb{E}(L)$. However, when $k$ has characteristic zero in general we cannot take $Z=\mathbb{E}(L)$, by Keel $[12, \S 3]$. Although $Z$ is not unique some choice can be calculated for any given $X, L$. It is interesting to see whether the theorem holds if $L$ is only $\mathbb{R}$-Cartier.

The augmented base locus of log divisors. Let $(X, B)$ be a projective pair over an algebraically closed field $k$ and $A$ a nef and big $\mathbb{R}$-divisor such that $L=K_{X}+B+A$ is nef. The locus $\mathbf{B}_{+}(L)$ is closely related to the geometry of $X$. In Section 6 we recall some results and pose some questions concerning such loci.

Acknowledgements. I would like to thank Mircea Mustaţă and Karl Schwede for discussions related to Section 6, and thank the referee for the valuable comments and corrections. This work was supported by a grant of the Leverhulme Trust.

\section{Preliminaries}

2.1. Growth of functions. Let $h: \mathbb{Z} \rightarrow \mathbb{Z}$ be a function. We say that the upper growth of $h$ is like $m^{d}$ (resp. at most like $m^{d}$ ) if

$$
0<\limsup _{m \rightarrow+\infty} \frac{h(m)}{m^{d}}<+\infty
$$

(resp. $\left.\lim \sup _{m \rightarrow+\infty} \frac{h(m)}{m^{d}}<+\infty\right)$.

2.2. Divisors. Let $X$ be a scheme. The group of Cartier divisors on $X$ is denoted by $\operatorname{Div}(X)$. Recall that an $\mathbb{R}$-Cartier divisor (resp. $\mathbb{Q}$-Cartier divisor) is an element of $\operatorname{Div}(X) \otimes_{\mathbb{Z}} \mathbb{R}$ (resp. $\left.\operatorname{Div}(X) \otimes_{\mathbb{Z}} \mathbb{Q}\right)$. Such a divisor can be represented as $L=\sum l_{i} L_{i}$ where $l_{i} \in \mathbb{R}$ (resp. $l_{i} \in \mathbb{Q}$ ) and $L_{i}$ are Cartier divisors but this representation is not unique. Two $\mathbb{R}$-Cartier divisors $L, L^{\prime}$ are $\mathbb{R}$-linearly equivalent (resp. $\mathbb{Q}$-linearly equivalent) if $L-L^{\prime}=\sum a_{i} N_{i}$ where $a_{i} \in \mathbb{R}$ (resp. $a_{i} \in \mathbb{Q}$ ) and $N_{i}$ are Cartier divisors linearly equivalent to zero. We denote the equivalence by $L \sim_{\mathbb{R}} L^{\prime}$ (resp. $L \sim_{\mathbb{Q}} L^{\prime}$ ). Note that each $\mathbb{R}$-Cartier divisor $L=\sum_{1}^{r} l_{i} L_{i}$, determines an $\mathbb{R}$-line bundle $\mathcal{L}:=\mathcal{O}_{X}\left(L_{1}\right)^{l_{1}} \otimes \cdots \otimes \mathcal{O}_{X}\left(L_{r}\right)^{l_{r}}$ in $\operatorname{Pic}(X) \otimes_{\mathbb{Z}} \mathbb{R}$. 
Let $f: X^{\prime} \rightarrow X$ be a morphism from another scheme $X^{\prime}$ and $L$ an $\mathbb{R}$-Cartier divisor on $X$. If $f$ is flat, then one can define the pullback $f^{*} L$ by taking the pullback of the local defining equations of $L$ (eg, when $X$ is over a field $k$ and $f$ is induced by base change of $k$ to a field $k^{\prime}$ ). However, we cannot define the pullback $f^{*} L$ in general if $f$ is not flat, although we can define the pullback of the associated $\mathbb{R}$-line bundle $\mathcal{L}$ (i.e. the element in $\operatorname{Pic}(X) \otimes_{\mathbb{Z}} \mathbb{R}$ corresponding to $L$ ), say $\mathcal{L}^{\prime}:=f^{*} \mathcal{L}$. If $\mathcal{L}^{\prime}$ is the $\mathbb{R}$-line bundle associated to some $\mathbb{R}$-Cartier divisor $L^{\prime}$ (eg, this is the case if $X^{\prime}$ is an integral scheme, or if $X^{\prime}$ is projective over a field [14]), then we can define $L^{\prime}=f^{*} L$ but this determines $L^{\prime}$ only up to $\mathbb{R}$-linear equivalence. In this paper, when we talk about pullback of divisors, either the morphism is flat or that pullback is defined up to $\mathbb{R}$-linear equivalence (eg, the restriction $\left.L\right|_{V}$ in Definition 1.3).

Now assume $X$ is a projective scheme over a field $k$. An $\mathbb{R}$-Cartier divisor $L$ on $X$ is:

- nef if $L \cdot C \geq 0$ for every curve $C \subseteq X$ (a curve is an integral closed subscheme of dimension one);

- ample if $L \sim_{\mathbb{R}} \sum l_{i} L_{i}$ with $l_{i}>0$ and $L_{i}$ ample Cartier divisors;

- effective if $L=\sum l_{i} L_{i}$ with $l_{i} \geq 0$ and $L_{i}$ effective Cartier divisors;

- big if $L \sim_{\mathbb{R}} A+D$ where $A$ is an ample $\mathbb{R}$-Cartier divisor and $D$ is an effective $\mathbb{R}$-Cartier divisor;

- semi-ample if $L=\sum l_{i} L_{i}$ where $0 \leq l_{i} \in \mathbb{R}$ and $L_{i}$ are base point free Cartier divisors.

2.3. The operator $\langle-\rangle$. Let $X$ be a projective scheme over a field $k$ and $L$ be an $\mathbb{R}$-Cartier divisor with an expression $L \sim_{\mathbb{R}} \sum t_{i} A_{i}$ as in 1.2. Let $\pi: X^{\prime} \rightarrow X$ be a morphism such that the pullbacks $A_{i}^{\prime}:=\pi^{*} A_{i}$ are defined up to linear equivalence and assume $A_{i}$ are very ample, eg $\pi$ is obtained by base change as in 2.7 below or $\pi$ is a closed embedding. Then we get the expression $L^{\prime}:=\pi^{*} L \sim_{\mathbb{R}} \sum t_{i} A_{i}^{\prime}$ which we can use to define $\left\langle m L^{\prime}\right\rangle$. Here $L^{\prime}$ is defined up to $\mathbb{R}$-linear equivalence. It is clear that $\left\langle m L^{\prime}\right\rangle \sim \pi^{*}\langle m L\rangle$.

For a coherent sheaf $\mathcal{F}$ on $X$, we often use the notation $\mathcal{F}\langle m L\rangle$ instead of $\mathcal{F}(\langle m L\rangle)$.

2.4. Pairs. A pair $(X, B)$ over a field $k$ consists of a normal quasi-projective variety over $k$ and a Weil $\mathbb{R}$-divisor $B$ with coefficients in $[0,1]$ such that $K_{X}+B$ is $\mathbb{R}$-Cartier where $K_{X}$ is the canonical divisor. The pair is $k l t$ if for every projective birational morphism $f: Y \rightarrow X$ from a normal variety the coefficients of $B_{Y}$ are all $<1$ where $K_{Y}+B_{Y}=f^{*}\left(K_{X}+B\right)$.

2.5. Fujita vanishing theorem. This is a generalization of Serre vanishing theorem. Let $X$ be a projective scheme over a field $k, A$ an ample Cartier divisor, and $\mathcal{F}$ a coherent sheaf on $X$. Then there is a number $m_{0}$ such that $h^{i}(\mathcal{F}(m A+L))=0$ for any $i>0, m>m_{0}$, and nef Cartier divisor $L$ [11],[13, Theorem 1.4.35]. 
The augmented base locus of real divisors over arbitrary fields 5

2.6. Restriction to a hyperplane section. Let $X$ be a projective scheme over a field $k, A$ an effective Cartier divisor, and $\mathcal{F}$ a coherent sheaf on $X$. Tensoring $\mathcal{F}$ with the exact sequence

$$
0 \rightarrow \mathcal{O}_{X}(-A) \rightarrow \mathcal{O}_{X} \rightarrow \mathcal{O}_{A} \rightarrow 0
$$

gives a sequence

$$
0 \rightarrow \mathcal{F}(-A) \rightarrow \mathcal{F} \rightarrow \mathcal{F} \otimes \mathcal{O}_{A} \rightarrow 0
$$

which is often not exact on the left. However, if $A$ (considered as a closed subscheme) does not contain any of the finitely many associated points of $\mathcal{F}$, then the latter sequence is also exact on the left. If $A$ is very ample and if $k$ is infinite, then it is a well-known fact that after changing $A$ up to linear equivalence we can make sure that $A$ does not contain any associated point of $\mathcal{F}$.

2.7. Base loci and base change. Let $X$ be a projective scheme over a field $k$ and let $L$ be a Cartier divisor on $X$. Recall that the base locus of $L$ is defined as

$$
\mathrm{Bs}|L|=\left\{x \in X \mid \alpha \text { vanishes at } x \text { for every } \alpha \in H^{0}\left(\mathcal{O}_{X}(L)\right)\right\} .
$$

As pointed out earlier we consider Bs $|L|$ (and other loci) with the reduced structure. Recall that $\mathbf{B}(L)=\bigcap_{m \in \mathbb{N}} \operatorname{Bs}|m L|$. If $n, n^{\prime} \in \mathbb{N}$, then each section $\alpha \in H^{0}\left(\mathcal{O}_{X}(n L)\right)$ gives a section $\alpha^{\otimes n^{\prime}} \in H^{0}\left(\mathcal{O}_{X}\left(n^{\prime} n L\right)\right)$ hence Bs $\left|n^{\prime} n L\right| \subseteq$ Bs $|n L|$. In particular, $\mathbf{B}(L)=\operatorname{Bs}|m L|$ for every sufficiently divisible $m>0$.

Assume that $k \subseteq k^{\prime}$ is a field extension and $X^{\prime}$ is the scheme obtained by base change to $k^{\prime}$. Let $\pi: X^{\prime} \rightarrow X$ be the corresponding morphism. Since $\pi$ is flat, we can define the pullback $L^{\prime}=\pi^{*} L$. Since

$$
H^{0}\left(\mathcal{O}_{X}\left(L^{\prime}\right)\right)=H^{0}\left(\mathcal{O}_{X}(L)\right) \otimes_{k} k^{\prime}
$$

we can see that $\pi^{-1} \operatorname{Bs}|L|=\operatorname{Bs}\left|L^{\prime}\right|$. This in turn implies that $\pi^{-1} \mathbf{B}(L)=$ $\mathbf{B}\left(L^{\prime}\right)$.

Now assume that $L$ is $\mathbb{R}$-Cartier with a given expression $L \sim_{\mathbb{R}} \sum t_{i} A_{i}$ as in 1.2. As pointed out in 2.3, $\left\langle m L^{\prime}\right\rangle \sim \pi^{*}\langle m L\rangle$ hence $\pi^{-1} \mathbf{B}_{+}(L)=\mathbf{B}_{+}\left(L^{\prime}\right)$.

With a little more work we can also see that $\pi^{-1} \mathbb{E}(L) \supseteq \mathbb{E}\left(L^{\prime}\right)$. Indeed, let $V^{\prime}$ be a component of $\mathbb{E}\left(L^{\prime}\right)$, let $W$ be the closure of $\pi\left(V^{\prime}\right)$, and let $W^{\prime}$ be the scheme obtained from $W$ by base change. If $\left.L\right|_{W}$ is big then $\left.L\right|_{W} \sim_{\mathbb{R}} A_{W}+D_{W}$ where $A_{W}$ is ample and $D_{W}$ is effective. But then $\left.L^{\prime}\right|_{W^{\prime}} \sim_{\mathbb{R}} A_{W^{\prime}}^{\prime}+D_{W^{\prime}}^{\prime}$ where $A_{W^{\prime}}^{\prime}$ is ample and $D_{W^{\prime}}^{\prime}$ is effective. Now $V^{\prime} \nsubseteq D_{W^{\prime}}^{\prime}$ otherwise $W \subseteq D_{W}$ which is not possible. Thusby restricting to $V^{\prime}$ we get $\left.L^{\prime}\right|_{V^{\prime}} \sim_{\mathbb{R}} A_{V^{\prime}}^{\prime}+D_{V^{\prime}}^{\prime}$ which means that $\left.L^{\prime}\right|_{V^{\prime}}$ is big, a contradiction. Therefore $\pi^{-1} \mathbb{E}(L) \supseteq \mathbb{E}\left(L^{\prime}\right)$. Thusif in some situation we want to show that $\mathbf{B}_{+}(L) \subseteq \mathbb{E}(L)$, then it is enough to show that $\mathbf{B}_{+}\left(L^{\prime}\right) \subseteq \mathbb{E}\left(L^{\prime}\right)$ because $\pi$ is surjective.

\section{The AUgmented Base LOCUS IS WELL-DEFINED}

In this section, we show that the augmented base locus as defined in Definition 1.2 is well-defined. We also show that the definition agrees with 1.1 and the one in [8]. 
Lemma 3.1. Let $X$ be a projective scheme over a field $k$ and $L$ an $\mathbb{R}$-Cartier divisor with a given expression $L \sim_{\mathbb{R}} \sum t_{i} A_{i}$ as in 1.2. Then $\mathbf{B}_{+}(L)$ as defined in 1.2 satisfies the following assertions:

(1) $\mathbf{B}_{+}(L)$ does not depend on the choice of $A$ nor the expression $L \sim_{\mathbb{R}}$ $\sum t_{i} A_{i}$

(2) for any positive rational number $s$ we have $\mathbf{B}_{+}(s L)=\mathbf{B}_{+}(L)$;

(3) $\mathbf{B}_{+}(L)=\bigcap \mathbf{B}(L-H)$ where $H$ runs over all ample $\mathbb{R}$-Cartier divisors so that $L-H$ is $\mathbb{Q}$-Cartier;

(4) if $L$ is $\mathbb{Q}$-Cartier then $\mathbf{B}_{+}(L)$ coincides with the one defined in 1.1.

Proof. (1) First we show that $\mathbf{B}_{+}(L)$ is independent of the choice of $A$. Indeed let $G$ be any other ample Cartier divisor. Assume $x \notin \bigcap_{m \in \mathbb{N}} \mathbf{B}(\langle m L\rangle-A)$. Then

$$
x \notin \mathbf{B}(\langle m L\rangle-A)=\mathbf{B}\left(\sum\left\lfloor m t_{i}\right\rfloor A_{i}-A\right)
$$

for some $m>0$. Thus $x \notin \mathbf{B}\left(\sum l\left\lfloor m t_{i}\right\rfloor A_{i}-l A\right)$ for any sufficiently large $l>0$. Since

$$
\sum\left\lfloor l m t_{i}\right\rfloor A_{i}-\sum l\left\lfloor m t_{i}\right\rfloor A_{i}
$$

is zero or ample, $x \notin \mathbf{B}\left(\sum\left\lfloor l m t_{i}\right\rfloor A_{i}-l A\right)$, and since $l A$ is sufficiently ample,

$$
x \notin \mathbf{B}\left(\sum\left\lfloor l m t_{i}\right\rfloor A_{i}-G\right)=\mathbf{B}(\langle\operatorname{lm} L\rangle-G) .
$$

This shows that

$$
\bigcap_{m \in \mathbb{N}} \mathbf{B}(\langle m L\rangle-A) \supseteq \bigcap_{m \in \mathbb{N}} \mathbf{B}(\langle m L\rangle-G) .
$$

The opposite inclusion $\subseteq$ can be proved similarly hence $\mathbf{B}_{+}(L)$ is independent of $A$.

Now we show that $\mathbf{B}_{+}(L)$ is independent of the expression $L \sim_{\mathbb{R}} \sum t_{i} A_{i}$. Indeed assume that $L \sim_{\mathbb{R}} \sum t_{i}^{\prime} A_{i}^{\prime}$ is another expression. Redefining the indexes we can assume that $A_{i}^{\prime}=A_{i}$. Let $A=\sum A_{i}+G$ with $G$ ample. Assume that

$$
x \notin \bigcap_{m \in \mathbb{N}} \mathbf{B}\left(\sum\left\lfloor m t_{i}\right\rfloor A_{i}-A\right) .
$$

Then $x \notin \mathbf{B}\left(\sum\left\lfloor m t_{i}\right\rfloor A_{i}-A\right)$ for some $m$ hence $x \notin \mathbf{B}\left(\sum l\left\lfloor m t_{i}\right\rfloor A_{i}-l A\right)$ for any sufficiently large $l>0$. Arguing as above we can show that $x \notin$ $\mathbf{B}\left(\sum\left\lfloor l m t_{i}\right\rfloor A_{i}-A\right)$. Writing $l m t_{i}=\left\lfloor l m t_{i}\right\rfloor+u_{i}$ and $l m t_{i}^{\prime}=\left\lfloor l m t_{i}^{\prime}\right\rfloor+u_{i}^{\prime}$, we see that

is ample hence $x \notin \mathbf{B}\left(\sum\left\lfloor l m t_{i}^{\prime}\right\rfloor A_{i}-G\right)$ so

$$
\begin{gathered}
\left(\sum\left\lfloor l m t_{i}^{\prime}\right\rfloor A_{i}-G\right)-\left(\sum\left\lfloor l m t_{i}\right\rfloor A_{i}-A\right) \\
\sim_{\mathbb{R}} \sum u_{i} A_{i}-\sum u_{i}^{\prime} A_{i}+A-G
\end{gathered}
$$

$$
x \notin \bigcap_{m \in \mathbb{N}} \mathbf{B}\left(\sum\left\lfloor m t_{i}^{\prime}\right\rfloor A_{i}-G\right) .
$$

In other words,

$$
\bigcap_{m \in \mathbb{N}} \mathbf{B}\left(\sum\left\lfloor m t_{i}\right\rfloor A_{i}-A\right) \supseteq \bigcap_{m \in \mathbb{N}} \mathbf{B}\left(\sum\left\lfloor m t_{i}^{\prime}\right\rfloor A_{i}-G\right) .
$$


The augmented base locus of real divisors over arbitrary fields 7

The opposite inclusion $\subseteq$ can be proved similarly bearing in mind that we are free to change $A$ and $G$.

(2) It is enough to treat the case when $s \in \mathbb{N}$. It is obvious that $\mathbf{B}_{+}(s L) \supseteq$ $\mathbf{B}_{+}(L)$. Assume that $x \notin \mathbf{B}_{+}(L)$. Let $A=\sum A_{i}$. Then $x \notin \mathbf{B}(\langle m L\rangle-A)$ for some $m$ hence $x \notin \mathbf{B}(s\langle m L\rangle-s A)$. Since $\langle m s L\rangle-A-(s\langle m L\rangle-s A)$ is ample or zero, we see that $x \notin \mathbf{B}(\langle m s L\rangle-A)$ which implies that $x \notin \mathbf{B}_{+}(s L)$. That is, $\mathbf{B}_{+}(s L) \subseteq \mathbf{B}_{+}(L)$.

(3) For each $m>0$,

$$
\mathbf{B}(\langle m L\rangle-A)=\mathbf{B}\left(m L-m H_{m}\right)=\mathbf{B}\left(L-H_{m}\right)
$$

for some ample $\mathbb{R}$-Cartier divisor $H_{m}$. Thus $\mathbf{B}_{+}(L) \supseteq \bigcap \mathbf{B}(L-H)$. Conversely assume $x \notin \bigcap \mathbf{B}(L-H)$. Then $x \notin \mathbf{B}(L-H)$ for some $H$. Since $L-H$ is assumed to be $\mathbb{Q}$-Cartier, $m L-m H$ is Cartier for some sufficiently divisible $m>0$. Since $m H$ is sufficiently ample,

$$
\mathbf{B}(m L-m H) \supseteq \mathbf{B}(\langle m L\rangle-A)
$$

hence $x \notin \mathbf{B}(\langle m L\rangle-A)$ which implies that $x \notin \mathbf{B}_{+}(L)$.

(4) We can write $L \sim_{\mathbb{Q}} \sum t_{i} A_{i}$ with all the $t_{i}$ rational numbers. Pick $s \in \mathbb{N}$ so that $s L$ is Cartier, $s t_{i}$ are all integers, and $s L \sim \sum s t_{i} A_{i}$. Then by (2) and (1) we have

$$
\begin{gathered}
\mathbf{B}_{+}(L)=\mathbf{B}_{+}(s L)=\bigcap_{m \in \mathbb{N}} \mathbf{B}\left(\sum\left\lfloor m s t_{i}\right\rfloor A_{i}-s A\right) \\
=\bigcap_{m \in \mathbb{N}} \mathbf{B}(m s L-s A)=\bigcap_{m \in \mathbb{N}} \mathbf{B}(m L-A) .
\end{gathered}
$$

But this is the same as $\mathbf{B}_{+}(L)$ in Definition 1.1.

\section{Growth of Cohomology}

The next lemma is similar to [6, Lemma 2.2].

Lemma 4.1. Let $X$ be a scheme projective over a field $k$. Assume that $L$ is an $\mathbb{R}$-Cartier divisor with a given expression $L \sim_{\mathbb{R}} \sum t_{i} A_{i}$ as in 1.2. Let $\mathcal{F}$ be a coherent sheaf on $X$, let $Y$ be its support, and $d=\operatorname{dim} Y$. Then the upper growth of $h^{0}(\mathcal{F}\langle m L\rangle)$ is at most like $m^{d}$.

Proof. By 2.3, we can extend $k$ hence assume it is infinite. Let $t$ be a positive integer such that $t_{i} \leq t$ for every $i$. By 2.6, we can change the $A_{i}$ up to linear equivalence so that for each $m>0$,

$$
\mathcal{F}\langle m L\rangle=\mathcal{F}\left(\sum\left\lfloor m t_{i}\right\rfloor A_{i}\right) \subseteq \mathcal{F}\left(m t \sum A_{i}\right) .
$$

Thusby replacing $L$ with $t \sum A_{i}$ it is enough to assume that $L$ is an effective very ample Cartier divisor. But then for $m$ sufficiently large $h^{0}(\mathcal{F}(m L))$ coincides with the Hilbert polynomial of $\mathcal{F}$ with respect to $L$ which is a polynomial of degree $m^{d}$. 
Lemma 4.2. Let $X$ be a scheme projective over a field $k$. Assume that $A$ is a very ample Cartier divisor and $\mathcal{F}$ is a coherent sheaf on $X$. Then we have $h^{0}(\mathcal{F}) \leq h^{0}(\mathcal{F}(A))$.

Proof. By extending $k$ we can assume $k$ is infinite. By 2.6, we can change $A$ up to linear equivalence so that $\mathcal{F} \subseteq \mathcal{F}(A)$ which implies the claim.

Lemma 4.3. Let $X$ be an integral scheme of dimension d projective over a field $k$. Assume that $L$ is an $\mathbb{R}$-Cartier divisor with a given expression $L \sim_{\mathbb{R}} \sum t_{i} A_{i}$ as in 1.2. Then the following are equivalent:

(1) the upper growth of $h^{0}\left(\mathcal{O}_{X}\langle m L\rangle\right)$ is like $m^{d}$;

(2) for some coherent sheaf $\mathcal{F}$, the upper growth of $h^{0}(\mathcal{F}\langle m L\rangle)$ is like $m^{d}$;

(3) for any coherent sheaf $\mathcal{F}$ whose support is equal to $X$, the upper growth of $h^{0}(\mathcal{F}\langle m L\rangle)$ is like $m^{d}$;

(4) $L$ is big.

Proof. (1) $\Longrightarrow$ (4): Let $A$ be an effective ample Cartier divisor. Considering the exact sequence

$$
0 \rightarrow \mathcal{O}_{X}(\langle m L\rangle-A) \rightarrow \mathcal{O}_{X}\langle m L\rangle \rightarrow \mathcal{O}_{A}\langle m L\rangle \rightarrow 0
$$

and applying Lemma 4.1, we deduce that the upper growth of $h^{0}\left(\mathcal{O}_{X}(\langle m L\rangle-A)\right)$ is like $m^{d}$. In particular, $\langle m L\rangle-A \sim D$ for some effective Cartier divisor $D$. Therefore, $m L \sim_{\mathbb{R}} A^{\prime}+D$ for some ample $\mathbb{R}$-Cartier divisor $A^{\prime}$ because $m L-\langle m L\rangle$ is zero or ample. Thus $L$ is big.

$(4) \Longrightarrow(3):$ By definition, $L \sim_{\mathbb{R}} A+D$ where $A$ is an ample $\mathbb{R}$-Cartier divisor and $D$ is an effective $\mathbb{R}$-Cartier divisor. Let $l \in \mathbb{N}$. Then for each $m \in \mathbb{N}$, $\langle m l L\rangle=m\langle l L\rangle+C_{m}$ for some $C_{m}$ which is zero or very ample. By Lemma 4.2,

$$
h^{0}(\mathcal{F}(m\langle l L\rangle)) \leq h^{0}(\mathcal{F}\langle m l L\rangle) .
$$

Moreover, if $l$ is large enough, then $\langle l L\rangle$ is big. Thusby replacing $L$ with $l L$ for some large $l$ and then replacing $L$ with $\langle L\rangle$ allows us to assume that $L$ is Cartier and $\langle m L\rangle=m L$ for each $m>0$. By replacing $A, D$ we can assume that $L \sim_{\mathbb{Q}} A+D$ and that $A, D$ are $\mathbb{Q}$-Cartier. Replacing $L, A, D$ with multiples we can assume $L \sim A+D$, that $A, D$ are Cartier, and $A$ is very ample.

First assume that $\mathcal{F}$ is generated by global sections. Each global section corresponds to a morphism $\mathcal{O}_{X} \rightarrow \mathcal{F}$. Since $X$ is integral, the morphism is injective if and only if its image is not torsion. Therefore if $\alpha_{1}, \ldots, \alpha_{r}$ form a basis of $H^{0}(\mathcal{F})$ and if $\phi_{i}: \mathcal{O}_{X} \rightarrow \mathcal{F}$ corresponds to $\alpha_{i}$, then $\phi_{i}$ is injective for at least one $i$ otherwise $\mathcal{F}$ would be torsion which is not possible as the support of $\mathcal{F}$ is equal to $X$. Therefore,

$$
h^{0}\left(\mathcal{O}_{X}(m A)\right) \leq h^{0}\left(\mathcal{O}_{X}(m L)\right) \leq h^{0}(\mathcal{F}(m L))
$$

which implies that the upper growth of $h^{0}(\mathcal{F}(m L))$ is like $m^{d}$.

Now we deal with the general case when $\mathcal{F}$ is not necessarily generated by global sections. Tensor $\mathcal{F}$ with $\mathcal{O}_{X}(-D) \rightarrow \mathcal{O}_{X}$ to get $\mathcal{F}(-D) \rightarrow \mathcal{F}$ and let $\mathcal{K}$ and $\mathcal{M}$ be its kernel and image respectively. Then we get exact sequences

$$
0 \rightarrow \mathcal{K}(m L) \rightarrow \mathcal{F}(m L-D) \rightarrow \mathcal{M}(m L) \rightarrow 0
$$




\section{The augmented base locus of real divisors over arbitrary fields 9}

and

$$
0 \rightarrow \mathcal{M}(m L) \rightarrow \mathcal{F}(m L) \rightarrow \mathcal{F}(m L) \otimes \mathcal{O}_{D} \rightarrow 0 .
$$

Note that the support of $\mathcal{K}$ is inside $D$, so the upper growth of $h^{0}(\mathcal{K}(m L))$ is at most like $m^{d-1}$ by Lemma 4.1. On the other hand, replacing $L, A, D$ with multiples we can assume $\mathcal{F}(A)$ is generated by global sections. Thus by the last paragraph, the upper growth of

$$
h^{0}(\mathcal{F}(m L-D))=h^{0}(\mathcal{F}(A)((m-1) L))
$$

is like $m^{d}$. This implies the upper growth of $h^{0}(\mathcal{M}(m L))$ is like $m^{d}$ which in turn implies the upper growth of $h^{0}(\mathcal{F}(m L))$ is like $m^{d}$.

$(3) \Longrightarrow(2)$ : Obvious.

$(2) \Longrightarrow(1)$ : There is a filtration

$$
0=\mathcal{F}_{0} \subset \mathcal{F}_{1} \subset \cdots \subset \mathcal{F}_{n}=\mathcal{F}
$$

of coherent sheaves such that for each $0<j \leq n$, there exist a closed embedding $f: S \rightarrow X$ of an integral scheme $S$ and an ideal sheaf $\mathcal{J} \subset \mathcal{O}_{S}$ such that $\mathcal{F}_{j} / \mathcal{F}_{j-1} \simeq f_{*} \mathcal{J}$ (cf. The stacks project [18], section on dévissage of coherent sheaves). Let $j$ be the smallest number such that the upper growth of $h^{0}\left(\mathcal{F}_{j}\langle m L\rangle\right)$ is like $m^{d}$. Let $f: S \rightarrow X$ and $\mathcal{J}$ be the corresponding embedding and ideal sheaf so that $\mathcal{F}_{j} / \mathcal{F}_{j-1} \simeq f_{*} \mathcal{J}$. Then from the exact sequence

$$
0 \rightarrow H^{0}\left(\mathcal{F}_{j-1}\langle m L\rangle\right) \rightarrow H^{0}\left(\mathcal{F}_{j}\langle m L\rangle\right) \rightarrow H^{0}\left(f_{*} \mathcal{J}\langle m L\rangle\right)
$$

we deduce that the upper growth of $h^{0}\left(f_{*} \mathcal{J}\langle m L\rangle\right)$ is like $m^{d}$. By Lemma 4.1, $\operatorname{dim} S=d$, hence $S=X$. But then the upper growth of $h^{0}\left(\mathcal{O}_{X}\langle m L\rangle\right)$ is like $m^{d}$.

Proposition 4.4. Let $X$ be a projective scheme over a field $k$. Assume that $L$ is a nef $\mathbb{R}$-Cartier divisor with a given expression $L \sim_{\mathbb{R}} \sum t_{i} A_{i}$ as in 1.2. Let $\mathcal{F}$ be a coherent sheaf on $X$, let $Y$ be its support, and $e=\operatorname{dim} Y$. Then

(1) the upper growth of $h^{0}(\mathcal{F}\langle m L\rangle)$ is at most like $m^{e}$;

(2) the upper growth of $h^{i}(\mathcal{F}\langle m L\rangle)$ is at most like $m^{e-i}$ for any $i$;

(3) the upper growth of $h^{0}(\mathcal{F}\langle m L\rangle)$ is like $m^{e}$ if and only if $\left.L\right|_{Z}$ is big for some component $Z$ of $Y$ with $\operatorname{dim} Z=e$.

Proof. (1) This follows from Lemma 4.1.

(2) We do induction on $e$. By extending $k$ we can assume that it is infinite. Choose an effective sufficiently ample Cartier divisor $H$ and let $A=2 H$. For each $m>0$ we can write $\langle m L\rangle \sim_{\mathbb{R}} m L-\sum u_{i} A_{i}$ where $u_{i} \in[0,1)$ depend on $m$. Since $H$ is sufficiently ample and $L$ is nef,

$$
\langle m L\rangle+H \sim_{\mathbb{R}} m L-\sum u_{i} A_{i}+H
$$

is ample. Then by Fujita vanishing $(2.5)$ we get $h^{i}(\mathcal{F}(\langle m L\rangle+A))=0$ for every $m>0$ and $i>0$. By 2.6, we can choose $A$ so that the sequence

$$
0 \rightarrow \mathcal{F} \rightarrow \mathcal{F}(A) \rightarrow \mathcal{F} \otimes \mathcal{O}_{A}(A) \rightarrow 0
$$


is exact. The dimension of the support of $\mathcal{F} \otimes \mathcal{O}_{A}(A)$ is $e-1$. Now using the exact sequence

$$
H^{i-1}\left(\mathcal{F} \otimes \mathcal{O}_{A}(\langle m L\rangle+A)\right) \rightarrow H^{i}(\mathcal{F}(\langle m L\rangle)) \rightarrow H^{i}(\mathcal{F}(\langle m L\rangle+A))=0
$$

for $i>0$, and induction on $e$ we get the result.

(3) As in the proof of Lemma 4.3, there is a filtration

$$
0=\mathcal{F}_{0} \subset \mathcal{F}_{1} \subset \cdots \subset \mathcal{F}_{n}=\mathcal{F}
$$

of coherent sheaves such that for each $0<j \leq n$, there exist a closed embedding $f: S \rightarrow X$ of an integral scheme $S$ and an ideal sheaf $\mathcal{J} \subset \mathcal{O}_{S}$ such that $\mathcal{F}_{j} / \mathcal{F}_{j-1} \simeq f_{*} \mathcal{J}$

Assume that the upper growth of $h^{0}(\mathcal{F}\langle m L\rangle)$ is like $m^{e}$. Let $j$ be minimal with the property that the upper growth of $h^{0}\left(\mathcal{F}_{j}\langle m L\rangle\right)$ is like $m^{e}$. Let $f: S \rightarrow$ $X$ and $\mathcal{J}$ be the corresponding embedding and ideal sheaf so that $\mathcal{F}_{j} / \mathcal{F}_{j-1} \simeq$ $f_{*} \mathcal{J}$. Then the upper growth of $h^{0}\left(f_{*} \mathcal{J}\langle m L\rangle\right)$ is like $m^{e}$.Thusin particular $\mathcal{J} \neq 0$ and since $S$ is integral the support of $f_{*} \mathcal{J}$ is equal to $S$. Moreover, since the upper growth of $h^{0}\left(f_{*} \mathcal{J}\langle m L\rangle\right)$ is like $m^{e}$, Lemma 4.1 shows that $\operatorname{dim} S \geq e$. On the other hand, $S$ is a subset of $Y$ because $\left.\mathcal{F}\right|_{X \backslash Y}=0$ and because of the surjection $\mathcal{F}_{j} \rightarrow f_{*} \mathcal{J}$. Thus $\operatorname{dim} S \leq e$, hence $\operatorname{dim} S=e$. Now, by Lemma 4.3, $\left.L\right|_{S}$ is big and so we can take $Z=S$.

Conversely, assume that there is a component $Z$ of $Y$ of dimension $e$ such that $\left.L\right|_{Z}$ is big. In the filtration above, let $j$ be the smallest number such that $Z$ is a component of the support of $\mathcal{F}_{j}$. Then $Z$ is a subset of the support of the corresponding $f_{*} \mathcal{J}$ hence $Z \subseteq S$ which in turn implies that $Z=S$ because $e=\operatorname{dim} Z \leq \operatorname{dim} S \leq e$. It is then enough to show that the upper growth of $h^{0}\left(f_{*} \mathcal{J}\langle m L\rangle\right)$ is like $m^{e}$ because of the exact sequence

$$
0 \rightarrow H^{0}\left(\mathcal{F}_{j-1}\langle m L\rangle\right) \rightarrow H^{0}\left(\mathcal{F}_{j}\langle m L\rangle\right) \rightarrow H^{0}\left(f_{*} \mathcal{J}\langle m L\rangle\right) \rightarrow H^{1}\left(\mathcal{F}_{j-1}\langle m L\rangle\right)
$$

and the fact that the upper growth of $h^{1}\left(\mathcal{F}_{j-1}\langle m L\rangle\right)$ is at most like $m^{e-1}$ by (2). Now apply Lemma 4.3.

\section{Proof of MAin ReSUlts}

Proof. (of Theorem 1.4) By Noetherian induction we can assume that the theorem already holds for any closed subscheme of $X$ not equal to $X$.

Step 1. We deal with the first equality in the theorem. By definition, $\mathbf{B}_{+}(L) \subseteq \mathbf{B}(\langle n L\rangle-A)$ for any $n>0$. Moreover, there are positive integers $m_{1}, \ldots, m_{r}$ such that

$$
\mathbf{B}_{+}(L)=\mathbf{B}\left(\left\langle m_{1} L\right\rangle-A\right) \cap \cdots \cap \mathbf{B}\left(\left\langle m_{r} L\right\rangle-A\right)
$$

If $n=l m_{i}$ for some positive integer $l$, then $\langle n L\rangle-l\left\langle m_{i} L\right\rangle$ is zero or ample hence

$$
\mathbf{B}(\langle n L\rangle-A) \subseteq \mathbf{B}\left(l\left\langle m_{i} L\right\rangle-A\right) \subseteq \mathbf{B}\left(l\left\langle m_{i} L\right\rangle-l A\right)=\mathbf{B}\left(\left\langle m_{i} L\right\rangle-A\right)
$$

Therefore $\mathbf{B}_{+}(L)=\mathbf{B}(\langle n L\rangle-A)$ if each $m_{i} \mid n$. 


\section{The augmented base locus of real divisors over arbitrary fields 11}

For the second equality: for any fixed $n^{\prime}>0$ divisible by all the $m_{i}$ and any sufficiently divisible $l>0$ we have

$$
\begin{gathered}
\mathbf{B}_{+}(L)=\mathbf{B}\left(\left\langle n^{\prime} L\right\rangle-A\right)=\mathrm{Bs}\left|l\left\langle n^{\prime} L\right\rangle-l A\right| \supseteq \operatorname{Bs}\left|\left\langle l n^{\prime} L\right\rangle-A\right| \\
\supseteq \mathbf{B}\left(\left\langle l n^{\prime} L\right\rangle-A\right)=\mathbf{B}_{+}(L) .
\end{gathered}
$$

Now take $n=l n^{\prime}$.

Step 2. The rest of the proof will be devoted to showing $\mathbf{B}_{+}(L)=\mathbb{E}(L)$. It is obvious that $\mathbf{B}_{+}(L) \supseteq \mathbb{E}(L)$ so we will focus on the reverse inclusion. If $\left.L\right|_{Z}$ is not big for every component $Z$ of $X$ (with the reduced induced structure), then $\mathbf{B}_{+}(L) \subseteq \mathbb{E}(L)=X$. Thuswe may assume that there is a component $Z$ such that $\left.L\right|_{Z}$ is big. Pick such a $Z$ with maximal dimension, say $e$. Let $Y$ be the union of the other components, again with the induced reduced structure.

There are coherent ideal sheaves $\mathcal{I}, \mathcal{J} \subset \mathcal{O}_{X}$ such that the support of $\mathcal{I}$ is inside $Z$ but the support of $\mathcal{O}_{X} / \mathcal{I}$ is inside $Y$, and the support of $\mathcal{J}$ is inside $Y$ but the support of $\mathcal{O}_{X} / \mathcal{J}$ is inside $Z$ (cf. [18], section on dévissage of coherent sheaves). Let $Y^{\prime}, Z^{\prime}$ be the closed subschemes defined by $\mathcal{I}, \mathcal{J}$ respectively. On $Z \backslash Y$ we have $\mathcal{J}=0$ and $\mathcal{O}_{Z^{\prime}}=\mathcal{O}_{X}$. Thus the reduced scheme associated to $Z^{\prime}$ is nothing but $Z$. Similarly, one shows that the reduced scheme associated to $Y^{\prime}$ is $Y$. By construction, on $Z \backslash Y$ we have $\mathcal{O}_{Z^{\prime}}=\mathcal{O}_{X}$ and $\mathcal{I}=\mathcal{O}_{X}$, and on $Y \backslash Z$ we have $\mathcal{I}=0$.

Step 3. We would like to find sections of $\mathcal{O}_{X}(\langle n L\rangle-A)$ which vanish on $Y^{\prime}$ but not on $Z^{\prime}$. Let $\mathcal{I} \rightarrow \mathcal{O}_{Z^{\prime}}$ be the composition $\mathcal{I} \hookrightarrow \mathcal{O}_{X} \rightarrow \mathcal{O}_{Z^{\prime}}$ and let $\mathcal{K}, \mathcal{L}$ be its kernel and image respectively. Similarly, let $\mathcal{L} \rightarrow \mathcal{O}_{Z}$ be the composition $\mathcal{L} \hookrightarrow \mathcal{O}_{Z^{\prime}} \rightarrow \mathcal{O}_{Z}$ and let $\mathcal{N}, \mathcal{M}$ be its kernel and image respectively. Then, by Step 2, on $Z \backslash Y$ we have $\mathcal{L}=\mathcal{O}_{Z^{\prime}}$ and $\mathcal{M}=\mathcal{O}_{Z}$, and on $Y \backslash Z$ we have $\mathcal{L}=\mathcal{M}=0$. Therefore the support of $\mathcal{L}, \mathcal{M}, \mathcal{I}$ are all equal to $Z$, and the support of $\mathcal{K}, \mathcal{N}$ are subsets of $Z$.

Now we have the exact sequences

$$
0 \rightarrow \mathcal{K}(\langle n L\rangle-A) \rightarrow \mathcal{I}(\langle n L\rangle-A) \rightarrow \mathcal{L}(\langle n L\rangle-A) \rightarrow 0
$$

and

$$
0 \rightarrow \mathcal{N}(\langle n L\rangle-A) \rightarrow \mathcal{L}(\langle n L\rangle-A) \rightarrow \mathcal{M}(\langle n L\rangle-A) \rightarrow 0 .
$$

By Proposition 4.4, the upper growth of $h^{0}(\mathcal{M}(\langle n L\rangle-A))$ is like $n^{e}$ but the upper growth of $h^{1}(\mathcal{N}(\langle n L\rangle-A))$ is at most like $n^{e-1}$. On the other hand, again by Proposition 4.4, the upper growth of $h^{0}(\mathcal{L}(\langle n L\rangle-A))$ is like $n^{e}$ but the upper growth of $h^{1}(\mathcal{K}(\langle n L\rangle-A))$ is at most like $n^{e-1}$. Therefore for infinitely many $n>0$ we can lift a nonzero section of $\mathcal{M}(\langle n L\rangle-A)$ to a section of $\mathcal{L}(\langle n L\rangle-A)$ and in turn to a section of $\mathcal{I}(\langle n L\rangle-A)$. In other words, there is a section $\alpha \in H^{0}(\mathcal{I}(\langle n L\rangle-A))$ whose restriction to $Z$ is nonzero. Since $\mathcal{I}$ is the ideal sheaf of $Y^{\prime}, \alpha$ vanishes on $Y^{\prime}$ when considered as a section of $\mathcal{O}_{X}(\langle n L\rangle-A)$ via the injection $\mathcal{I}(\langle n L\rangle-A) \rightarrow \mathcal{O}_{X}(\langle n L\rangle-A)$. 
Step 4. From now on we consider $\alpha$ as a section of $\mathcal{O}_{X}(\langle n L\rangle-A)$. We can think of $\alpha$ as a morphism $\mathcal{O}_{X} \rightarrow \mathcal{O}_{X}(\langle n L\rangle-A)$ such that if we tensor this with $\mathcal{O}_{Z}$ then we obtain a nonzero morphism. Let $\alpha_{1}:=\alpha$ and let $\mathcal{T}_{1}$ be the kernel of $\alpha_{1}$. Let $\alpha_{2}$ be the composition

$$
\mathcal{O}_{X} \rightarrow \mathcal{O}_{X}(\langle n L\rangle-A) \rightarrow \mathcal{O}_{X}(2\langle n L\rangle-2 A) \rightarrow \mathcal{O}_{X}(\langle 2 n L\rangle-2 A)
$$

where the first morphism is $\alpha_{1}$, the second one is obtained by tensoring $\alpha_{1}$ with $\mathcal{O}_{X}(\langle n L\rangle-A)$, and the third one comes from the choice of an injective morphism $\mathcal{O}_{X} \rightarrow \mathcal{O}_{X}(\langle 2 n L\rangle-2\langle n L\rangle)$ (which exists because $\langle 2 n L\rangle-2\langle n L\rangle$ is zero or very ample) and tensoring it with $\mathcal{O}_{X}(2\langle n L\rangle-2 A)$.

Let $\mathcal{T}_{2}$ be the kernel of $\alpha_{2}$. Obviously, $\mathcal{T}_{1} \subseteq \mathcal{T}_{2}$. Inductively we can define $\alpha_{i}$ to be the composition

$\mathcal{O}_{X} \rightarrow \mathcal{O}_{X}(\langle(i-1) n L\rangle-(i-1) A) \rightarrow \mathcal{O}_{X}(\langle(i-1) n L\rangle+\langle n L\rangle-i A) \rightarrow \mathcal{O}_{X}(\langle i n L\rangle-i A)$

where the first map is $\alpha_{i-1}$, the second map is obtained by tensoring $\alpha_{1}$ with $\mathcal{O}_{X}(\langle(i-1) n L\rangle-(i-1) A)$, and third one is obtained from the choice of an injective morphism $\mathcal{O}_{X} \rightarrow \mathcal{O}_{X}(\langle i n L\rangle-\langle(i-1) n L\rangle-\langle n L\rangle)$. Again it is obvious that $\mathcal{T}_{i-1} \subseteq \mathcal{T}_{i}$

Step 5. By the Noetherian property, there is $r$ such that $\mathcal{T}_{r}=\mathcal{T}_{r+1}=\cdots$. Since $\alpha_{1}$ restricted to $Z$ is nonzero and since $Z$ is integral, we can make sure that the restriction of each $\alpha_{i}$ to $Z$ is also nonzero: indeed if $U \subset Z$ is a small nonempty open set, then the restriction to $U$ of each map in the definition of $\alpha_{i}$ is an isomorphism. Therefore each $\alpha_{i}$ is nonzero hence $\mathcal{T}_{r} \subsetneq \mathcal{O}_{X}$.

Now tensor $\alpha_{r}$ with $\mathcal{O}_{X}(-\langle r n L\rangle+r A)$ and let $\mathcal{E}$ be its image in $\mathcal{O}_{X}$. Then we get the exact sequence

$$
0 \rightarrow \mathcal{T}_{r}(-\langle r n L\rangle+r A) \rightarrow \mathcal{O}_{X}(-\langle r n L\rangle+r A) \rightarrow \mathcal{E} \rightarrow 0
$$

Let $E$ be the closed subscheme defined by $\mathcal{E}$, that is, $E$ is the zero subscheme of $\alpha_{r}$. Note that since $\left.\alpha_{r}\right|_{Z}$ is nonzero, $Z \nsubseteq E$.

We will argue that $\mathbf{B}(\langle m L\rangle-A) \subseteq E$ if $m>0$ is sufficiently divisible. By construction, $\alpha_{r}$ does not vanish outside $E$ hence $\mathbf{B}(\langle r n L\rangle-r A) \subseteq E$. If $m=\operatorname{lrn}$, then

$$
\begin{gathered}
\mathbf{B}(\langle m L\rangle-A) \subseteq \mathbf{B}(l\langle r n L\rangle-A) \subseteq \mathbf{B}(l\langle r n L\rangle-l A) \\
=\mathbf{B}(\langle r n L\rangle-A) \subseteq \mathbf{B}(\langle r n L\rangle-r A) \subseteq E
\end{gathered}
$$

Step 6. We will assume that $r \gg 0$. Consider the exact sequence $0 \rightarrow \mathcal{T}_{r}(\langle m L\rangle-\langle r n L\rangle+r A-a A) \rightarrow \mathcal{O}_{X}(\langle m L\rangle-\langle r n L\rangle+r A-a A) \rightarrow \mathcal{E}(\langle m L\rangle-a A) \rightarrow 0$.

Since $\mathcal{T}_{r}$ does not depend on $r \gg 0$, by Fujita vanishing, we may assume that

$$
H^{i}\left(\mathcal{T}_{r}(\langle m L\rangle-\langle r n L\rangle+r A-a A)\right)=0
$$

and

$$
H^{i}\left(\mathcal{O}_{X}(\langle m L\rangle-\langle r n L\rangle+r A-a A)\right)=0
$$




\section{The augmented base locus of real divisors over arbitrary fields 13}

for any $i>0, m>r n$, and $a \in\{0,1\}$. Therefore $H^{i}(\mathcal{E}(\langle m L\rangle-a A))=0$ if $i>0, m \gg 0$, and $a \in\{0,1\}$ (in this proof we only need to consider $a=1$ but in the proof of Theorem 1.5 we need to take $a=0$ ).

On the other hand, we have the exact sequence

$$
0 \rightarrow \mathcal{E}(\langle m L\rangle-a A) \rightarrow \mathcal{O}_{X}(\langle m L\rangle-a A) \rightarrow \mathcal{O}_{E}(\langle m L\rangle-a A) \rightarrow 0
$$

from which we obtain the exact sequence

$$
H^{0}\left(\mathcal{O}_{X}(\langle m L\rangle-a A)\right) \rightarrow H^{0}\left(\mathcal{O}_{E}(\langle m L\rangle-a A)\right) \rightarrow H^{1}(\mathcal{E}(\langle m L\rangle-a A))=0
$$

if $m \gg 0$ and $0 \leq a \leq 1$.

Step 7. From the expression $L \sim_{\mathbb{R}} \sum t_{i} A_{i}$ we obtain the expression $\left.L\right|_{E} \sim_{\mathbb{R}}$ $\left.\sum t_{i} A_{i}\right|_{E}$. The restriction $\left.L\right|_{E}$ (resp. $\left.A_{i}\right|_{E}$ ) is defined up to $\mathbb{R}$-linear equivalence (resp. linear equivalence). For each $m>0$ we get $\left.\langle m L\rangle\right|_{E}=\left\langle\left. m L\right|_{E}\right\rangle$. Taking $a=1$ in Step 6, recalling that $\mathbf{B}(\langle m L\rangle-A) \subseteq E$ if $m>0$ is sufficiently divisible, and using Step 1, we deduce that

$$
\begin{gathered}
\mathbf{B}_{+}(L)=\mathbf{B}(\langle m L\rangle-A)=\mathrm{Bs}|\langle m L\rangle-A| \\
=\mathrm{Bs}\left|\left\langle\left. m L\right|_{E}\right\rangle-A\right|_{E} \mid=\mathbf{B}\left(\left\langle\left. m L\right|_{E}\right\rangle-\left.A\right|_{E}\right)=\mathbf{B}_{+}\left(\left.L\right|_{E}\right)
\end{gathered}
$$

for any sufficiently divisible $m>0$.

On the other hand, it is easy to see that $\mathbb{E}\left(\left.L\right|_{E}\right) \subseteq \mathbb{E}(L)$ : indeed, if $V$ is a component of $\mathbb{E}\left(\left.L\right|_{E}\right)$, then $\left.\left(\left.L\right|_{E}\right)\right|_{V}$ is not big, so $\left.L\right|_{V}$ is not big, hence $V \subseteq \mathbb{E}(L)$. Finally using the Noetherian induction and the above results we get

$$
\mathbb{E}(L) \subseteq \mathbf{B}_{+}(L)=\mathbf{B}_{+}\left(\left.L\right|_{E}\right)=\mathbb{E}\left(\left.L\right|_{E}\right) \subseteq \mathbb{E}(L)
$$

which in particular implies that $\mathbf{B}_{+}(L)=\mathbb{E}(L)$.

Proof. (of Theorem 1.5) We may assume that the theorem holds for every closed subscheme of $X$ other than $X$ itself. Moreover, by replacing $L$ with a multiple we can assume that it is Cartier. If $\mathbb{E}(L)=X$, the theorem is trivial. We thus assume this is not the case. Let $E$ be the subscheme constructed in Step 5 of the proof of Theorem 1.4. We showed that if $m \gg 0$, the map

$$
H^{0}\left(\mathcal{O}_{X}(m L)\right) \rightarrow H^{0}\left(\mathcal{O}_{E}(m L)\right)
$$

is surjective (by taking $a=0$ ). Moreover, we showed that $\mathbb{E}\left(\left.L\right|_{E}\right)=\mathbb{E}(L)$. Since

$$
\mathbf{B}(L) \subset \mathbf{B}_{+}(L)=\mathbb{E}(L) \subseteq E
$$

we have $\mathbf{B}(L)=\mathbf{B}\left(\left.L\right|_{E}\right)$. Thus $L$ is semi-ample if and only if $\left.L\right|_{E}$ is semiample. Since the theorem already holds for $E$ by assumption, there is a closed subscheme $Z$ of $E$ whose reduction is $\mathbb{E}\left(\left.L\right|_{E}\right)$ and such that $\left.L\right|_{E}$ is semi-ample if and only if $\left.L\right|_{Z}$ is semi-ample. Now $L$ is semi-ample if and only if $\left.L\right|_{Z}$ is semi-ample. 


\section{The AUgmented BASE LOCUS OF LOG DIVISORS}

Assume that $X$ is a normal projective variety of dimension $d$ over an algebraically closed field $k$, and that $B, A \geq 0$ are $\mathbb{R}$-divisors. Moreover, suppose $A$ is nef and big and $L=K_{X}+B+A$ is nef.

Theorem 6.1. Assume $L^{d}=0$. Then $\mathbf{B}_{+}(L)=X$ is covered by rational curves $C$ with $L \cdot C=0$.

The theorem was proved by Cascini-Tanaka-Xu [7] and independently by $\mathrm{M}^{\mathrm{c}}$ Kernan, when $k$ has positive characteristic. A short proof of this in any characteristic was given in [1]. Now if $L^{d}>0$, what can we say about $\mathbf{B}_{+}(L)$ ? For example, is it again covered by rational curves intersecting $L$ trivially? We give a couple of examples to shed some light on this question.

Example 6.2 Let $E$ be an elliptic curve over an algebraically closed field $k$ and let $X=\mathbb{P}\left(\mathcal{O}_{E} \oplus \mathcal{O}_{E}(1)\right)$. The surjection $\mathcal{O}_{E} \oplus \mathcal{O}_{E}(1) \rightarrow \mathcal{O}_{E}$ defines a section of the projection $X \rightarrow E$ whose image will be denoted by $E$ again. Moreover, there is a birational contraction $X \rightarrow Z$ which contracts only $E$. Let $B=E$ and $A$ be the pullback of a sufficiently ample divisor on $Z$. Let $L=K_{X}+B+A$. By construction, $\mathbf{B}_{+}(L)=\mathbb{E}(L)=E$ which is not covered by rational curves but at least it is covered by curves intersecting $L$ trivially.

Example 6.3 There is a well-known example of a smooth projective surface $S$ over an algebraically closed field $k$, which is ruled over an elliptic curve $Z$, containing a curve $M$ (a section of $S \rightarrow Z$ ) such that the Kodaira dimension of $M$ as a divisor on $S$ is zero, $K_{S}+2 M \sim 0$, and if $M \cdot C=0$ for some curve $C$ then $C=M$ (see Shokurov [17, Example 1.1] for such an example). Let $X=\mathbb{P}\left(\mathcal{O}_{S} \oplus \mathcal{O}_{S}(1)\right)$. The surjection $\mathcal{O}_{S} \oplus \mathcal{O}_{S}(1) \rightarrow \mathcal{O}_{S}$ defines a section of the projection $\pi: X \rightarrow S$ whose image will be denoted by $S$ again. Moreover, there is a birational contraction $X \rightarrow Z$ which contracts only $S$ to a point. Let $B=S+3 \pi^{*} M$ and let $A$ be the pullback of a sufficiently ample divisor on $Z$. Let $L=K_{X}+B+A$. Then $\mathbf{B}_{+}(L)=\mathbb{E}(L) \subseteq S$. Moreover, since

$$
\left.L\right|_{S}=\left.\left(K_{X}+S+3 \pi^{*} M+A\right)\right|_{S} \sim K_{S}+3 M \sim M
$$

is not big, $\mathbf{B}_{+}(L)=\mathbb{E}(L)=S$. But there is no family of curves $C$ covering $S$ with the property $L \cdot C=0$.

These examples show that we need to put some reasonably strong condition on $X, B, A$ to be able to say something interesting about $\mathbf{B}_{+}(L)$.

Question 6.4. Assume that $(X, B)$ is a projective klt pair over an algebraically closed field $k$ and $A$ a nef and big $\mathbb{R}$-divisor. Assume that $L=K_{X}+B+A$ is nef and that $L^{d}>0$. Is it true that $\mathbf{B}_{+}(L)$ is covered by rational curves $C$ with $L \cdot C=0$ ?

Assume that $k=\mathbb{C}$. Then $L$ in the question is semi-ample by the base point free theorem hence it defines a contraction $X \rightarrow Y$. Moreover, it is well-known that the fibres of $X \rightarrow Y$ are covered by rational curves. Note that $\mathbf{B}_{+}(L)$ is nothing but the union of the fibres of $X \rightarrow Y$. 


\section{The augmented base locus of real divisors over arbitrary fields 15}

Now assume that $k$ has characteristic $p>5$ and $\operatorname{dim} X \leq 3$. One can show that $L$ is again semi-ample (if $\operatorname{dim} X=2$, this holds for any $p$ [19]). We sketch the proof. Since $A$ is nef and big, we can change the situation so that it is ample [1, Lemma 8.2]. Using boundedness of the length of extremal rays [12],[1, 3.3] one can show that $L=\sum r_{i}\left(K_{X}+B_{i}+A_{i}\right)$ where $r_{i}>0, \sum r_{i}=1, B_{i}, A_{i}$ are effective $\mathbb{Q}$-divisors, $A_{i}$ is ample, $\left(X, B_{i}\right)$ is klt, and $K_{X}+B_{i}+A_{i}$ is nef and big. Now applying [1, Theorem 1.4],[20] each $K_{X}+B_{i}+A_{i}$ is semi-ample hence $L$ is also semi-ample. Thus $L$ defines a contraction $X \rightarrow Y$. In particular, $\mathbf{B}_{+}(L)$ is covered by a family of curves intersecting $L$ trivially. Using the results of [1] it does not seem hard to prove that the fibres of $X \rightarrow Y$ are actually covered by rational curves.

Assume that $k$ has positive characteristic and $\operatorname{dim} X \geq 4$. It seems hard to answer the question in this case because of the lack of resolution of singularities. However, if we replace the klt condition with strongly $F$-regular, then it is likely that one can actually answer the question.

\section{REFERENCES}

[1] C. Birkar; Existence of flips and minimal models for 3-folds in char $p$. To appear in Annales scientifiques de l'ENS, arXiv:1311.3098.

[2] C. Birkar, Z. Hu; Log canonical pairs with good augmented base loci. Compositio Math. Volume 150 (2014), 579-592.

[3] S. Boucksom, S. Cacciola, A. F. Lopez; Augmented base loci and restricted volumes on normal varieties. Mathematische Zeitschrift 278 (2014), 979-985.

[4] S. Boucksom, J-P. Demailly, M. Păun, Th. Peternell; The pseudo-effective cone of a compact Kahler manifold and varieties of negative Kodaira dimension. J. Algebraic Geom. 22 (2013), 201-248.

[5] S. Cacciola, A. F. Lopez; Nakamaye's theorem on log canonical pairs. Annales de l'Institut Fourier 64 (2014), n. 6, 2283-2298.

[6] P. Cascini, J. McKernan, M. Mustaţă; The augmented base locus in positive characteristic. Proceedings of the Edinburgh Mathematical Society (Series 2), Volume 57, Issue 01, (2014), 79-87.

[7] P. Cascini, H. Tanaka, C. Xu; On base point freeness in positive characteristic. To appear in Annales scientifiques de l'ENS, arXiv:1305.3502v1.

[8] L. Ein, R. Lazarsfeld, M. Mustaţă, M. Nakamaye, M. Popa; Restricted volumes and base loci of linear series. Amer. J. Math., 131(3), (2009), 607-651.

[9] L. Ein, R. Lazarsfeld, M. Mustaţă, M. Nakamaye, M. Popa; Asymptotic invariants of base loci. Ann. Inst. Fourier (Grenoble) 56, (2006) 1701-1734.

[10] O. Fujino, H. Tanaka; On log surfaces. Proc. Japan Acad. Ser. A Math. Sci. Volume 88, Number 8 (2012), 109-114.

[11] T. Fujita; Vanishing theorems for semipositive line bundles. In Algebraic geometry (Tokyo/Kyoto, 1982), 519-528, Lecture Notes in Math. 1016, Springer, Berlin, 1983.

[12] S. Keel; Basepoint freeness for nef and big line bundles in positive characteristic. Annals of Math, Second Series, Vol. 149, No. 1 (1999), 253-286.

[13] R. Lazarsfeld; Positivity in algebraic geometry. I. Springer-Verlag (2004).

[14] Y. Nakai; Some fundamental lemmas on projective schemes. Trans. Amer. Math. So. 109 (1963), 296-302.

[15] M. Nakamaye; Base loci of linear series are numerically determined. Trans. Amer. Math. Soc. 355 (2002), 551-566.

[16] M. Nakamaye; Stable base loci of linear series. Math. Ann. 318 (2000), 837-847. 
[17] V.V. Shokurov; Complements on surfaces. Algebraic geometry, 10. J. Math. Sci. (New York) 102 (2000), no. 2, 3876-3932.

[18] The stacks project. Website: http://stacks.math.columbia.edu.

[19] H. Tanaka; Minimal models and abundance for positive characteristic log surfaces. To appear in Nagoya Math. J., arXiv:1201.5699v2.

[20] C. Xu; On base point free theorem of threefolds in positive characteristic. To appear in J. Inst. Math. Jussieu., arXiv:1311.3819.

DPMMS, Centre for Mathematical Sciences, Cambridge University, Wilberforce Road, Cambridge, CB3 0WB, UK

email: c.birkar@dpmms.cam.ac.uk 